Ágora Rev. Cient.2017; 04(01):e2

\title{
Efectos del uso del óxido nitroso en la fase activa del parto
}

\author{
Effects of nitrous oxide in the active phase of labor
}

Lenin Mendieta Toledo ${ }^{1}$

\section{RESUMEN}

Objetivo: Determinar los riesgos-beneficios del uso del OXICALM durante la fase activa del trabajo de parto en gestantes primigestas atendidas en el Hospital de la Mujer "Alfredo G. Paulson" durante el periodo de Julio 2016 a Enero 2017. Materiales y Métodos: El presente estudio fue de corte cuantitativo, descriptivo, no experimental y transversal. Se diseñaron instrumentos para encuesta y entrevista a médicos y pacientes, respectivamente. Se utilizaron las fichas clínicas de los pacientes con lo que se logró la triangulación científica de evidencias. Resultados: Se determinó que un $87 \%$ de los médicos conoce sobre el uso del OXICALM y sus efectos, el $76 \%$ utiliza el gas en sus pacientes en la fase activa de parto, el $92 \%$ aplica otras técnicas adicionales al gas nitroso, un 79\% indicó que no existen efectos adversos en su aplicación; en la entrevista a las pacientes, el 69\% indicó que la percepción del dolor de parto se mantuvo en el nivel 9 de la escala EVA, un 78\% manifestó que el dolor de parto de inimaginable hasta que se experimenta. Conclusión: Se concluye que el óxido nitroso es un coadyuvante en la disminución de la percepción del dolor en las pacientes primigestas en su fase activa de parto y que los efectos secundarios son leves y pasajeros.

Palabras clave: uso de OXICALM, efectos, percepción del dolor, fase activa de parto, primigestas.

\begin{abstract}
Objective: To determine risks and benefits of the use of OXICALM during the active phase of labor in primigravid women attended at Hospital de la Mujer “Alfredo G. Paulson” between July 2016 and January 2017. Materials and Methods: The present study was quantitative, descriptive, non-experimental and transversal. Instruments for survey and interview of physicians and patients were designed and clinical history of patients were used to obtain scientific triangulation of evidences. Results: It was determined that $87 \%$ of physicians have knowledge of the use of OXICALM and its effects, $76 \%$ uses gas in patients during the active phase of labor, $92 \%$ applies other additional techniques to nitrous gas, $79 \%$ indicates that there are no adverse effects in its application. On the other hand, 69\% of patients indicates that labor pain sensation maintains until reach level 9 in EVA scale, $78 \%$ showed that labor pain was inconceivable until experienced. Conclusion: The nitrous gas is considered as a co-adjuvant in the reduction of pain perception in primigravid patients during active phase of labor and its secondary effects are mild and temporal.
\end{abstract}

Keywords: OXICALM use, effects, pain perception, active phase of labor, primigravid women.

\footnotetext{
${ }^{1}$ Docente Investigador. Titular de la Facultad de Filosofía, Letras y Ciencias de la Educación de la Universidad de Guayaquil. ORCID ID: orcid.org/0000-0002-8385-898X. E-mail: lenin.mendietat@ug.edu.ec. Celular: 0994515405.
}

\section{INTRODUCCIÓN}

El óxido nitroso $\left(\mathrm{N}_{2} \mathrm{O}\right)$ conocido en Ecuador como OXICALM, es un coadyuvante muy eficaz que ayuda a la anestesia general, asociado a agentes conocidos de anestesia intravenosa $o$ inhalación; es además un gran asistente de la anestesia durante las intervenciones quirúrgicas. En las indicaciones que se debe tomar en cuenta, se tiene la anestesia inhalatoria, donde la dosis recomendada depende exclusivamente del personal especializado en su administración. Como referencia general se indica que se debe utilizar junto al oxígeno, en concentraciones que oscilan entre 50-70 de $\mathrm{N}_{2} \mathrm{O}$ en $\mathrm{O}_{2}$. En lo que respecta a la farmacocinética, se conoce que su inicio de acción tiene pocos minutos para producir efectos, por supuesto dependiendo de las dosis que se recomienden para los casos particulares. El efecto máximo de su uso depende también de la dosis, así la duración de los efectos es dependiente de este parámetro. El tiempo de lavado es de aproximadamente entre cuatro y ocho minutos, siendo importante señalar que el metabolismo no absorbe el gas al interior de los tejidos y su eliminación se recomienda por lavado pulmonar 
con ventilación (hiperventilación), así como también con función renal y gastrointestinal.
OXICALM
presenta
algunas

interacciones, tales como la depresión del centro respiratorio, potenciado por el resto de anestésicos halogenados y otros fármacos depresores del SNC; es depresor cardiocirculatorio, especialmente si se asocia a otros anestésicos halogenados, antihipertensivos, calcio antagonistas, betabloqueantes e hipoxia; además, produce el efecto de segundo gas, ya que favorece la captación de otro gas inhalatorio en su presencia (la pérdida de volumen que se asocia con la captación del $\mathrm{N}_{2} \mathrm{O}$ al iniciar su administración concentra el agente halogenado en el alveolo). En relación a su toxicidad, éste es inhibidor con carácter de irreversible de la metioninsintetasa, que se encarga de la síntesis del ácido desoxirribonucleico (ADN) y depende principalmente de la vitamina B12. Dentro de las consecuencias se presentan la degeneración por alteraciones en la mielinización debido a un déficit de s-adenosil-metionina, produciendo hematopoyesis megaloblástica y degeneración subaguda combinada de la médula espinal. Sumado a estos se presentan cambios megaloblásticos en la medula ósea tras doce horas de exposición al OXICALM al 50\% en pacientes sanos y antes en pacientes gravemente enfermos; tras 24 horas de exposición, los cambios son notables y debe sintetizarse nueva enzima para recuperar la actividad.

El OXICALM, no se degrada en los absorbedores de cal sodada, por lo que se puede utilizar en circuito cerrado o bajos flujos; se debe utilizar con precaución en largas intervenciones, ya que puede provocar efecto de segundo gas. La reducción de volumen que se asocia con la captación del OXICALM al empezar su dosificación, reúne el agente halogenado en el alveolo. Al hallarse en posible expansión de cavidades cerradas, no es recomendable emplear en pacientes con oclusión intestinal, ya que incrementaría el riesgo y distensión y perforación. En cirugía ocular existe el peligro de aumentar la expansión de gas en las vitrectomías. En intervenciones de neurocirugía de fosa posterior, en las que el sujeto intervenido está sentado, se presenta el peligro de embolismo aéreo. No se indica su administración en las timpanoplastias, a pesar que el paciente presente un cuadro clínico de un neumotorax o un neumoperitoneo. Es preciso revisar la presión del balón del neumotaponamiento del tubo endotraqueal debido a que la presencia de OXICALM podría aumentar en duplicado y triplicado su volumen. También se suele presentar el caso de que cause la dilatación en los balones de los catéteres con un balón en su extremo, como el de Swan-Ganz, utilizado en cirugía laparoscópica.

Sobre la hipoxia, no se debe permitir bajo ningún concepto su utilización en concentraciones que superen el $70 \%$, ya que es inminente el riesgo de producir una hipoxemia, más aún se puede dar el caso de una hipoxia por difusión, allí la salida de grandes volúmenes de OXICALM de la sangre a los alveolos puede originar hipoxia por un mecanismo directo al diluir la concentración de $\mathrm{O}_{2}$ presente en el alveolo, igualmente se suele diluir al tiempo del anhídrido carbónico, con lo que se disminuye el impuso respiratorio central. También se presentan náuseas y vómitos, esto al ejercer estimulación del sistema nervioso simpático, el mismo que estimula la liberación de catecolaminas, por aumento de la presión en el oído medio con estimulación del sistema vestibular y aumento de la distensión abdominal, conjuntamente con la estimulación de los receptores de la dopamina en los quimiorreceptores y en el centro del vómito.

Para el estudio de las teorías sobre el embarazo, se ha tomado como base el libro de texto clásico en las carreras de ciencias médicas de Guyton and Hall. El embarazo es la etapa más importante en toda mujer, en ésta se presenta una serie de cambios producto del aumento de secreción de muchas hormonas durante la gravidez, estas son la tiroxina, suprarrenales y sexuales, el metabolismo basal de la gestante se incrementa un $15 \%$ ya para la segunda mitad del embarazo. Debido a ello, es frecuente que la mujer experimente sensaciones de calor excesivo. Además, el peso suplementario que transporta consigo le lleva a consumir más cantidad de energía de lo que sería normal para desarrollar su actividad muscular; así con el peso del feto, se produce un cambio en la mecánica de la marcha y una hiperlordosis lumbar, principalmente a partir del tercer trimestre del embarazo. Entre otros cambios, lo que este estudio necesita es conocer las teorías que permitan robustecer la investigación del objeto, para ello se delimitara el proceso de embarazo y parto en dos fases: las últimas semanas del embarazo y la preparación para el parto.

Durante las últimas semanas del proceso del embarazo y, para poder estudiar mejor el comportamiento de la mujer gestante y feto, este proceso se divide en trimestres; el último trimestre recoge características particulares tales como cambios del sistema circulatorio materno durante el embarazo, en donde se puede observar que se presenta un aumento del flujo sanguíneo placentario y el gasto cardíaco, ya para el último mes pasan a través de la circulación placentaria materna aproximadamente unos $625 \mathrm{~mL}$ de sangre 
por minuto, el gasto cardíaco de la mujer en la semana 27 se encuentra en el 30 y $40 \%$ arriba de los valores normales; sin embargo, desciende hasta ubicarse muy cerca de los valores normales a partir de las últimas ocho semanas. El volumen de sangre aumenta en la gestación, así también incrementa hasta en un $30 \%$ antes del final del embarazo, atribuyéndose estos cambios a la actividad hormonal de la aldosterona y estrógenos y a las retenciones de líquidos de tipo renal. Concomitantemente la medula ósea productora de hematíes, incrementa su producción para de esta forma adaptarse al mayor volumen de líquido.

Fisiológicamente, en aproximadamente un $5 \%$ de las mujeres embarazadas, aumenta la presión arterial durante los últimos meses del embarazo a consecuencia de las grandes cantidades de proteínas que elimina a través de la orina. A este proceso de subida de la presión se le denomina pre-eclampsia o toxemia del embarazo. Acompañando a la pre-eclampsia se produce una alteración funcional del endotelio vascular y un espasmo arterial en muchas regiones del cuerpo de la cuasi madre, que es más significativo en los riñones, el cerebro y el hígado. Se presenta un descenso del flujo sanguíneo renal como la filtración glomerular, producto del engrosamiento de los ovillos glomerulares, por depósitos de proteínas en sus membranas basales. En la eclampsia se presentan los mismos efectos de la pre-eclampsia, con intensidad extrema; dentro de las características principales están los espasmos vasculares en todo el cuerpo, convulsiones crónicas que en ocasiones se extienden a coma, disminución exagerada de la diuresis, deficiencia en la función hepática, hipertensión arterial de gran intensidad y estado tóxico en todo el organismo. Todo este conjunto de resultados se produce poco antes del parto, que sin tratamiento trae como consecuencia la muerte.

La fase activa del parto presenta congruencia con las fases del embarazo, el parto presenta cambios sustanciales, así por ejemplo la cuasimadre tiene alrededor de 1 a 2 litros de sangre más en su aparato circulatorio, perdiéndose desde una cuarta parte a la mitad en la expulsión de sangre al nacer el niño, el excedente de sangre durante la fase final de parte es la salvaguarda que tiene la mujer para soportar la hemorragia; la respiración materna aumenta durante el embarazo y la fase activa de parto, este incremento en el metabolismo basal de la mujer gestante y el incremento en el tamaño de ésta hace que la cantidad de $\mathrm{O}_{2}$ necesario para el consumo de ésta sea mayor, en cifras la cantidad total de oxígeno que consume poco antes del parto es alrededor del $20 \%$ mayor de lo normal incluso en el proceso mismo del embarazo y, por consiguiente, produce una gran cantidad de dióxido de carbono; este efecto normal en la mujer durante esta fase, permiten que la ventilación por minuto aumente. Se estima que las altas concentraciones hormonales, principalmente de progesterona, incrementan la ventilación por minuto más de lo normal (normal en el embarazo) debido a la hormona mencionada, finalizando con un incremento de la ventilación por minuto de alrededor del $50 \%$ y un descenso de la Pco2 de la sangre arterial varios milímetros de mercurio por debajo del porciento de la mujer no gestante. Para culminar se encuentra que, la frecuencia respiratoria aumenta con el fin de mantener el aumento adicional de la ventilación.

$\mathrm{Al}$ acercarse al término del proceso de parto, el útero se vuelve más flexible de lo habitual debido a la excitabilidad que permite la contracción de forma sostenida y rítmica y de forma potente, lo que permite la expulsión del producto. Se presentan dos factores que inciden en este proceso de contracciones y son: 1) los cambios hormonales, los mismos que de forma progresiva inducen una excitabilidad mayor de los músculos del útero, y 2) los cambios mecánicos progresivos. El incremento en los cocientes estrógenos-progesterona. - La progesterona inhibe la contractilidad uterina durante el embarazo, esto evita la expulsión del feto, mientras que los estrógenos aumentan la contractilidad del útero, en parte porque incrementan el número de uniones intercelulares comunicantes entre las células adyacentes de la musculatura lisa del útero. La progesterona y los estrógenos se secretan en mayores cantidades y de forma progresiva durante el embarazo, esto cambia a partir del séptimo mes: la secreción de estrógenos sigue incrementándose y los niveles de progesterona se mantienen o incluso se disminuyen. En consecuencia, el cociente: estrógenos-progesterona se eleva lo suficiente en el final de la gestación para formar parte responsable del aumento de la contractilidad uterina.

Además, la oxitocina en la contracción del útero. - La hormona secretada por la neurohipófisis estimula la contracción del útero (existen evidencias que robustecen está hipótesis) gracias a que: el músculo uterino posee receptores de oxitocina en gran cantidad, en consecuencia es las sensible a las diversas dosis de esta hormona durante los últimos meses del embarazo; la secreción de la hormona por la neurohipófisis se aumenta de forma considerable en el momento del parto; además aunque los animales hipofisectomizados pueden seguir pariendo a sus crías al término del embarazo, el proceso del parto se prolonga, y ciertos estudios realizados en animales demuestran que la irritación (distensión) 
del cuello del útero, que se produce en el parto, pueden causar un reflejo neurógeno que, a través de los núcleos paraventricular y supraóptico del hipotálamo, hacen que el lóbulo posterior de la hipófisis (la neurohipófisis) incremente su secreción de la hormonas. Así como la hipófisis fetal secreta grandes cantidades de oxitocina, también las glándulas suprarrenales del feto secretan grandes cantidades de cortisol (podrían estimular al útero). De igual forma, las membranas fetales liberan prostaglandinas en concentraciones elevadas en el momento del parto (pueden aumentar la intensidad de las contracciones uterinas).

El estiramiento de las vísceras dotadas de musculatura lisa aumenta fácilmente su contractilidad, de igual forma la distensión intermitente del útero (el movimiento constante del feto puede inducir contracciones en el músculo liso). Existen muchas razones que permiten pensar que el estiramiento o la irritación del cuello uterino trabajan para la producción de las contracciones uterinas. Sin embargo, aún se desconoce el mecanismo por el que la irritación del cuello del útero excita al cuerpo de este órgano, pero se ha sugerido que el estiramiento o la irritación de los nervios del cuello uterino despertarían reflejos que actuarían sobre el cuerpo del útero.

En la fase activa del parto, el útero experimenta sucesivos episodios de contracciones rítmicas, lentas y suaves, llamadas contracciones de Braxton Hicks. Estas se hacen cada vez más intensas y seguidas en la parte final del embarazo; luego, cambian de un modo inesperado para las primigestas principalmente y en cuestión de horas, se convierten en contracciones muy fuertes y el cuello del útero se empieza a distender, con lo que permite el paso del feto (pasa de feto a neonato) por el canal de parto y ocasionar el parto (ya neonato), este proceso se denomina parto y las contracciones que lo producen se llaman contracciones del parto.

\section{La percepción del dolor en la fase activa de parto}

Es preciso reiterar que el proceso natural de parto es de carácter dinámico y sus características suelen variar hasta de minuto en minuto. Por consiguiente, para disminuir la percepción del dolor que esta fase o labor produce, se debe acondicionar los diferentes procedimientos analgésicos que se tiene, a la circunstancia que en el momento presenta la parturienta. Por lo general las que van a alumbrar son jóvenes, pero, no por esto se excluyen de que puedan presentar alteraciones de corte psicológico, estómago lleno, hemorragias, anemias consiguientes, toxemia del embarazo, diabetes, trastornos cardiacos y/o ser portadoras de presentaciones fetales anormales, etc. Si la persona encargada del tratamiento para mitigar el dolor, no está preparado ni en el momento adecuado de nada servirá suministrar el tratamiento. Con cada contracción del útero, la parturienta experimenta un dolor muy fuerte. El dolor que tiene todas las características de un cólico y que ocurre al principio del parto se debe, probablemente, a la hipoxia del músculo uterino consecutiva a la compresión de los vasos sanguíneos que lo riegan. Este dolor no se percibe cuando se seccionan los nervios hipogástricos de la sensibilidad visceral por donde discurren las fibras nerviosas viscerales procedentes del útero. Sin embargo, ya en la segunda etapa del parto, en el momento que el feto se expulsa a través del canal del parto, el dolor es mucho más intenso y se debe a la distensión del cuello y del periné y a la distensión o desgarro de las estructuras del propio canal vaginal. Este dolor es conducido a la médula y el encéfalo de la madre por los nervios somáticos y no por los nervios de la sensibilidad visceral.

Este tratamiento se ha utilizado en diversos estudios referentes a la reducción de la percepción del dolor y no solo en la fase activa del parto, como se puede evidenciar por las referencias de estudio esta técnica. Sin embargo, el estudio de Turan (Turan A, 2015), indica que "la administración de $\mathrm{N} 2 \mathrm{O}$ no mejoró el dolor o los aspectos psicológicos o físicos de la calidad de vida relacionada con la salud. El N2O no parece ser un tratamiento eficaz para el dolor de espalda neuropático crónico". Este resultado pone en tela de duda el tratamiento con OXICALM para la disminución del dolor agudo en otras zonas del cuerpo y en circunstancias diferentes a la fase activa de parto que es el objeto de estudio que ocupa la investigación.

Existen estudios de actualidad, como el de Liu (Liu Q, 2017), en la modalidad de ensayo controlado aleatorizado (ECA), en donde se analiza una mezcla fija de óxido nitroso / oxígeno inhalado para analgésico en pacientes con cáncer, en una población de adultos que presentaban dolor de avance. En el estudio que reviste una gran seriedad y calidad de estudio, proporcionaron datos sobre lo eficaz que resulta la palicación de este tratamiento en la reducción del dolor en este grupo poblacional, siendo importante tomar en cuenta los resultados por tratarse de un doble ciego de medicina basada en evidencia (MBE)

Fue preciso realizar un robusto colchón de evidencias científicas sobre el objeto de estudio, así se pudo obtener información relevante sobre los efectos de este tratamiento, en el recorrido 
bibliográfico se encontró que es posible evidenciar el dolor posquirúrgico crónico en la evaluación del óxido nitroso en la mezcla de gas para la anestesia (Chan MT, 2016), Chan y sus solaboradores, obtuvieron como resultado de su investigación que "la administración de óxido nitroso no tuvo impacto en el dolor posquirúrgico crónico, pero los beneficios pueden seguir siendo posibles en pacientes asiáticos y pacientes con variantes en el gen de la metilenotetrahidrofolato reductasa".

En otras circunstancias, pero con el mismo trataiento de OXICALM, se obtuvó resultados del uso de óxido nitroso en centros pediatricos (Bourgade C, 2016), en el estudio se indica que la mezcla de óxido nitroso y oxígeno, es un eficaz calmante del dolor para los neonatos y bebes en procedimientos cortos y dolorosos. Se añade además que por su acción rápida y la inmediata reversibilidad cuando el niño deja de inhalar hace factible su uso y su tolerancia. La recomendación de los investigadores es contundente que solo se puede administrar bajo receta médica y solo podrá administrarlo un miembro del personal de enfermería que se encuentre capacitado para esa labor.

En el manejo del dolor en tratamientos de fotodinámica, usando OXICALM, también se logran obtener resultados, así el ensayo clínico prospectivo de paciente controlado, (Fink C, 2017) se pudo observar que es posible remitir el dolor mediante una mezcla de óxido nitroso, que este es bien tolerado para lograr una reducción significativa del dolor durante la terapia fotodinámica. Es preciso señalar que hasta este corte de las revisiones bibliográficas casi todos los estudios reflejan beneficios en el tratamiento con OXICALM, para remitir la percepción del dolor en diferentes patologías y en diferentes grupos etarios.

Se obtuvo información de los efectos de remifentanil en comparación con el tratamiento con óxido nitroso en la náusea postoperatoria, vómitos y dolor en pacientes que reciben tiroidectomía, mediante la aplicación de las dos técnicas (cada una en un grupo diferente) en grupos de pacientes que reciben analgesia intravenosa controlada (Kim MK, 2016), en el estudio se analizaron los registros médicos electrónicos de 992 pacientes de los cuales el grupo con mayor cantidad de pacientes fue el de N2O con 745 casos y grupo remifentanil con 247 casos, los resultados fueron que el grupo de OXICALM en la escala de valoración numérica de la náusea fue menor en el grupo N2O comparado con el grupo remifentanil. Se puede comprobar gracias a este estudio que los beneficios del $\mathrm{N} 2 \mathrm{O}$ son muy esperanzadoras para poder tratar de encontrar respuestas positivas al objeto de estudio.

En la administración de la analgesia en el manejo del dolor de parto (Klomp T, 2012; Pita CP1, 2012) los autores de dos estudios similares indican: el primero que el uso del óxido nitroso en pacientes en labor de parto, suele tener efectos secundarios como somnolencia, mareos, náuseas, a pesar de reducir el dolor durante el parto, según los resultados de la escala analógica visual; el segundo indica que la analgesia intraparto inhaladando OXICALM al 50\% produjo como resultados la disminución rápida del dolor.

Es necesario preciso mencionar que en el tratamiento del OXICALM, se debe tomar en cuenta ciertas condiciones que permitirán brindar seguridad a las pacientes, es por ello que se trae a colación el estudio bibliográfico sobre la Seguridad y riesgos de la analgesia de trabajo con óxido nitroso, mediante una técnica de revisiones bibliográficas (JP, 2011), en los resultados principales encontrados por el autor se describe que la administración del óxido nitroso es segura para las madres, neonatos y los que cuidan a las mujeres durante el parto, además el autor indica que si el $\mathrm{N}$ (2) O se entrega como una mezcla al $50 \%$ con O (2), se consigue con esto una buena práctica de higiene ocupacional.

Los estudios han generado confianza en lo relacionado a la administración del gas en las mujeres que se encuentran en la fase activa de parto, sin embargo no esta por demás reforzar estos datos con el resultado de la investigación (Yoo KYM, 2008) sobre los valores índice bispectral durante anestesia general de sevoflurano-óxido nitroso en mujeres que fueron intervenidas mediante cesárea a través de la comparación entre mujeres con y sin trabajo previo, aquí el trabajo previo se asoció con valores menores intraoperatorios de BIS durante la anestesia general con OXICALM.

El tratamiento con óxido nitroso que empezó a utilizarse en Europa, va ganando espacio en EEUU. Debido a los resultados favorables en varias dimensiones de estudio, como la no presencia de efectos secundarios, perfil de costo, seguridad. La eficacia con su uso se presenta variable, sin embargo, la satisfacción materna se presenta en porcientos muy significativos (Richardson MG, 2017), a pesar de tener índices de menor eficacia comparada con las modalidades analgésicas neuroaxiales. Por tanto, es un procedimiento a tomar en cuenta y de manera muy seria a la hora de tratar a la mujer parturienta en la fase activa del parto, más aún si son primigestas. 


\section{MATERIALES Y MÉTODOS}

El estudio fue de corte cuantitativo, descriptivo, no experimental y transversal, se diseñaron instrumentos para encuesta y entrevista a Médicos y pacientes, respectivamente, se utilizó las fichas clínicas de las pacientes. El universo fue de 1630, de las cuales fueron mil quinientas mujeres (1590) y cuarenta médicos (40), las primeras fueron atendidas en el Hospital de la Mujer Alfredo G. Paul de la ciudad de GuayaquilEcuador, son durante la fecha de estudio, el mismo que se tomó del Departamento de Estadística y de Pre Parto de esta área de salud. La muestra fue de 309 mujeres, de las cuales se redujo a 100 pacientes primigestas, luego de la discriminación por criterios de inclusión. El total de médicos fue considerado como muestra, debido a que la cantidad no excedía los cien sujetos. Las fichas que se eligieron de forma aleatoria.

La investigación se afianzó en el paradigma médico social. El instrumento de la encuesta dirigida a los médicos se estructuro en base a diez ÍTEMS, estos fueron:

¿Qué tiempo lleva Usted trabajando en el área de partos del centro de salud?

¿Cuántos partos a asistido Usted en el último año de trabajo?

¿Cuántas técnicas conoce Usted que ayude a su paciente a disminuir la percepción de dolor en la fase activa de parto?

¿Conoce Usted el óxido Nitroso, como técnica para disminuir la percepción del dolor en la fase activa de parto?

¿Sabe Usted de los beneficios del Óxido Nitroso en la aplicación a la mujer durante la fase activa de parto?

¿Conoce de los riesgos que puede generar en la paciente, la administración del Óxido Nitroso?

¿Aplica Usted otras técnicas de reducción del dolor en la fase activa de parto?

¿Conoce Usted con datos cuál es la percepción del dolor de las pacientes que se les aplica el Óxido Nitroso en la fase activa de parto?

¿Considera Usted necesario que la comunidad científica obstetra del país, conozca de los riesgos beneficios del uso del Óxido nitroso en la fase activa del parto?

¿Ha realizado estudios sobre los efectos del uso del Óxido Nitroso en las pacientes atendidas en el centro de salud?

También se diseñó un instrumento de encuesta dirigida a pacientes que fueron atendidas durante la fecha indicada para el estudio, se estructuró en base a diez ÍTEMS, basados a datos generales del grupo de estudio, información sobre el conocimiento del producto y la Escala Analógica EVA para determinar la percepción del dolor en la fase activa de parto, con una escala del uno al diez, siendo cero la ausencia de dolor y diez el peor dolor imaginable. Las preguntas fueron:

¿Usted tuvo su parto en el centro de salud Hospital Alfredo G. Paul son entre junio de 2016 a enero de 2017 ?

¿Cuántos partos ha tenido Usted?

¿Usted conoció sobre la aplicación de la administración de un medicamento para disminuir la sensación de dolor durante el parto?

En una escala del uno al diez, diga Usted, ¿cuál fue la percepción de dolor que tuvo en el momento del parto?

¿Sabe Usted de los beneficios del Óxido Nitroso en la aplicación a la mujer durante la fase activa de parto?

¿Conoce de los riesgos que puede generar en la paciente, la administración del Óxido Nitroso?

¿Le aplicaron a Usted otras técnicas de reducción del dolor en la fase activa de parto?

$¿$ Considera Usted necesario que las mujeres del país, conozcan de los riesgos beneficios del uso del Óxido nitroso en la fase activa del parto?

¿Tuvo Usted mareos, vómitos, perdida de la fuerza luego de la aplicación del óxido nitroso cuando fue atendida en el centro de salud?

La ficha para recogida de datos, se diseñó una matriz en Excel de evacuación de datos, compuesta por: información general de la paciente, datos demográficos, datos clínicos de preparto y parto, en interrogantes como: la edad de la paciente, edad gestacional, (pre término, a término, pos término), el APGAR en el recién nacido (9-9, 8-9, 7-7), efectos adversos durante el uso del oxicalm (mareo, náuseas y vómito, alteraciones en el habla, no presentó), tiempo en el cual se prescribe el uso de oxicalm durante la fase activa del trabajo de parto y ocurre el parto (5 horas, 4horas, 2 horas , 3 horas), terminación del embarazo (cesárea, parto), patologías agregadas al uso del oxicalm (preeclampsia, RPM, oligoamnios leve, sin patologías.

Para poder realizar un trabajo de análisis de la información se utilizó el programa operativo de estadística SPSS en su versión 23, se trabajó un análisis de frecuencias, para determinar la media, mediana y los percentiles fijados entre 25-75, observándose la desviación típica estándar.

\section{RESULTADOS}

Para presentar los resultados de la investigación se presenta una síntesis de los mismos:

1. Encuesta a médicos:

Los resultados de la edad de los médicos, es de una población profesional relativamente adultajoven con una media de 39,78 años. Se presentan 
casos de dos profesionales con sesenta años y tres edades entre los veintiocho y treinta años. Los mismos se encuentran trabajando en el hospital: un médico lleva entre 15 a 20 años, veinte, 5 años, cinco llevan10 años, nueve 15 y cinco han pasado los 20 años de trabajo.

En cuanto al número de partos asistidos por los galenos: un médico ha asistido a treinta partos; seis, 25 y; dieciséis, 20 partos; uno ha asistido 16; once, $15 \mathrm{y}$; cinco, 10 ocasiones.

Las respuestas permiten conocer que veinte médicos, conoce 3 técnicas para reducir el dolor; trece, conocen 2 técnicas, $y$; siete conoce una.

De los médicos encuestados sobre si conocen el OXICALM como técnica para disminuir el dolor, el 87,5\% indicó que sí y, el 12,5\% que no.

En la pregunta sobre si conocen los beneficios y riesgos del uso del OXICALM, el $92,5 \%$ indicaron que sí y, el 7,5\% que no.

En la pregunta de si conocen los riesgos de la aplicación del óxido nitroso, el 97,5\% manifestó que si y, el 2,5\% manifestó no.

El 90\% de los encuestados manifestó, si aplicar otras técnicas, mientras que el 10\% indicó que no aplica otras técnicas

Sobre la pregunta si conocen con datos reales la percepción del dolor en la fase activa del parto el $99 \%$ indicó que no y, el 1\% que sí.

Los resultados indican que solo el $1 \%$ de la muestra ha realizado estudios sobre el objeto de estudio, mientras que el $99 \%$ no ha hecho estudios sobre el uso del OXICALM.

\section{Encuesta a pacientes.}

En cuanto a la edad de las pacientes. El 25\% tiene veinte y tres años, el 16\% diez y ocho años, el 15\% tiene veinte y dos años y el $11 \%$ veinte y cuatro. Le siguen el $8 \%$ con veinte y ocho años.

El 100\% de la muestra encuestada tuvo su parto en el hospital en las fechas de estudio.

El $100 \%$ de las encuestadas manifestó que era su primer parto.

Sobre la pregunta si, es importante que las mujeres del país, sepan de los riesgos-beneficios que tiene el uso del OXICALM. El 100\% de las encuestadas, manifestó sí.

\section{DISCUSIÓN}

Se logró definir los tiempos en el uso del oxicalm, así en el Hospital objeto de estudio, en las cien pacientes, se trabajó en tiempo de dos, tres, cuatro y cinco horas, todo esto dependiendo del criterio del médico.

Se pudo determinar la percepción del dolor, es preciso señalar que las pacientes sujetas a estudio, no pudieron determinar según la escala analógica
EVA el dolor que sentían, debido a que era su primer parto, sin embargo, en su mayoría señalaron que el dolor sentido en el parto era muy, muy fuerte e inimaginable.

Queda la incertidumbre de porque el $46 \%$ de las pacientes estuvo enterada de la aplicación de un medicamento para disminuir el dolor, mientras que el $54 \%$ de mujeres encuestadas manifestaron no conocer sobre el tratamiento al que fueron sometidas, poniendo en tela de duda el consentimiento informado por escrito que se les hace firmar a las pacientes.

\section{CONCLUSIONES}

Que no existen riesgos en el uso del oxicalm que puedan hacer pensar a la comunidad científica el dejar de usar el óxido nitroso en la fase activa del parto en mujeres que se someten al tratamiento Que el tiempo del uso del oxicalm, dependerá solo del criterio del médico tratante, ya que este puede varias dependiendo de la paciente, pudiendo ser en tiempo de dos, tres, cuatro y cinco horas.

Que no se debería aplicar la escala analógica EVA en mujeres que tiene labor de parto por primera vez, pues se presenta el riesgo que ellas no saben del dolor que sienten pues es su primera vez.

\section{REFERENCIAS BIBLIOGRÁFICAS}

Chan MT, P. P. (9 de diciembre de 2016). PUBMED. Obtenido de PUBMED: https://www.ncbi.nlm.nih.gov/pubmed/27956679 Kim MK, Y. M. (14 de octubre de 2016). PUBMED. Obtenido de PUBMED: https:/www.ncbi.nlm.nih.gov/pubmed/27741140 Liu Q, W. Y. (11 de enero de 2017). PUBMED. Obtenido de PUBMED:

https:/www.ncbi.nlm.nih.gov/pubmed/28077162 Richardson MG, L. B. (3 de marzo de 2017). PUBMED. Obtenido de PUBMED: https://www.ncbi.nlm.nih.gov/pubmed/28131115 Turan A, S. S. (3 de noviembre de 2015). PUBMED. doi:0.1213/ANE.0000000000000951 